\title{
Evaluation of the optical criteria for sessile serrated lesions of the colon: A prospective study on a colorectal cancer screening population
}

\section{(ㄷ)(우우}

\author{
Authors \\ Institutions \\ 1 Gastrointestinal Endoscopy Unit, Hospital Universitari i \\ Politècnic La Fe, Valencia, Spain \\ 2 Gastrointestinal Research Group, Health Research \\ Institute (IISLaFe), Hospital Universitari i Politècnic La Fe, \\ Valencia, Spain \\ 3 Pathology Department, Hospital Universitari i Politècnic \\ La Fe, Valencia, Spain
}

Marco Bustamante-Balén ${ }^{1,2}$, Carla Satorres ${ }^{1,2}$, David Ramos-Soler ${ }^{2,3}$, Maria García-Campos ${ }^{1}$, Noelia Alonso ${ }^{1,2}$, Marta Ponce $^{1,2}$, Lidia Argüello-Viudez ${ }^{1,2}$, Francisco Giner ${ }^{3}$, Jaime Ferrer-Lozano ${ }^{3}$, Vicente Pons-Beltrán ${ }^{1,2}$

submitted 2.6.2020

accepted after revision 28.9.2020

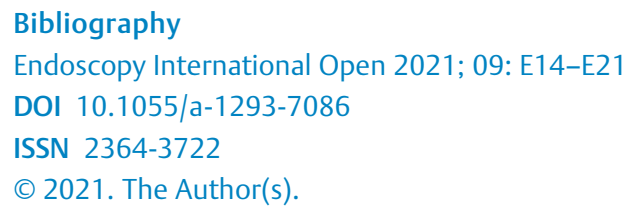
Commons Attribution-NonDerivative-NonCommercial License, permitting copying and reproduction so long as the original work is given appropriate credit. Contents may not be used for commecial purposes, or adapted, remixed, transformed or built upon. (https://creativecommons.org/licenses/by-nc-nd/4.0/)

\section{Corresponding author}

Marco Bustamante-Balén, MD, PhD, Gastrointestinal Endoscopy Unit, Hospital Universitari i Politècnic La Fe, Avda Fernando Abril Martorell, 106, Valencia 46026, Spain Fax: +34 961246278

bustamante_mar@gva.es

Supplementary material is available under https://doi.org/10.1055/a-1293-7086
ABSTRACT

Background and study aims We aimed to describe the presence and combination of Hazewinkel's optical diagnosis (OD) criteria for sessile serrated lesions (SSL), determining which lesion characteristics increase the probability of a correct OD, with a focus on diminutive lesions.

Patients and methods This was a prospective study describing the presence of Hazewinkel's OD criteria for SSL in lesions found in consecutive CRC screening colonoscopies. The presence of each OD criterion and their diagnostic combinations in SSL, related to the lesion's NBI International Colorectal Endoscopic (NICE) classification category, size, and location, were described. The presence of two or more optical criteria was considered diagnostic of SSL. The OD was compared to pathology as the gold standard.

Results Seventy-nine SSLs (5.6\%) were diagnosed. Cloudlike appearance was the most prevalent OD criterion (35, $44.3 \%)$. OD criteria were more frequently identified in NICE type $1, \geq 10 \mathrm{~mm}$, and proximal lesions. Only $26 \mathrm{SLLs}$ fulfilled the OD criteria (sensitivity $32.9 \%$, $95 \%$ Cl $29.1 \%-$ $36.7 \%$ ). The sensitivity for diminutive SSL was $14.7 \%$, (95\% Cl 11.9\%-17.6\%). Eighty-five lesions were optically diagnosed as SSL. However, only in 26 SSL was this the definitive diagnosis (positive predictive value $30.6 \%$, $95 \% \mathrm{Cl} 26.9 \%$ $34.3 \%$ ). Size $>5 \mathrm{~mm}$ and proximal location increased the probability of a correct diagnosis. The overall accuracy of the optical criteria was $92.0 \%(95 \% \mathrm{Cl}, 89.8 \%-94.2 \%)$.

Conclusions The Hazewinkel's optical criteria are not reliable for a positive diagnosis of SSL, particularly for diminutive lesions.

\section{Introduction}

Sessile serrated lesions (SSL) may be responsible for up to $35 \%$ of colorectal cancers (CRC) [1], through the so-called serrated pathway [2]. Because of their morphologic characteristics, they can be more easily missed or incompletely removed [3], and some data suggest that progression to cancer may be greatly accelerated when dysplasia is already present [4].
Therefore, SSL can be considered as an important group of premalignant lesions and responsible for, at least, a proportion of interval CRCs.

The resect and discard strategy relies on an accurate optical differentiation between neoplastic and non-neoplastic lesions [5]. Those diminutive lesions considered to be neoplastic should be resected and discarded, while rectal lesions classified as hyperplastic could be left in situ. Although in the first studies 
evaluating this strategy in practice only adenomas were considered premalignant [6], today it is obvious that SSL should be included in this category when implementing optical diagnosis (OD) of colorectal polyps [7]. Therefore, a positive and reliable method for the OD of SSL seems desirable.

Recently, four criteria for the OD of SSL using white light and narrow-band imaging (NBI) without magnification have been described. These are a cloud-like surface, indistinct borders, irregular shape, and dark spots, belonging to the so-called Hazewinkel's criteria ( $\mathbf{F i g . 1}$ ) [8]. The presence of two or more of these criteria allows for an OD of an SSL. Its combination with the NICE (NBI International Colorectal Endoscopic) classification conforming the WASP (Workgroup Serrated Polyps and Polyposis) classification has been shown to accurately discriminate between adenoma, hyperplastic polyp, and SSL in a study using still pictures [9]. One study has shown the ability of the WASP classification to fulfill the PIVI (Preservation and Incorporation of Valuable Endoscopic Innovations) criteria in real screening colonoscopies. However, the Hazewinkel's criteria seem to fall short in making a positive diagnosis of SSL because of reduced sensitivity and positive predictive values (PPV) [10]. The reason for this is not clear because little is known about how the specific Hazewinkel's criteria and their combinations are identified in colorectal lesions and specifically in SSL during real colonoscopies and how lesion characteristics influence the accuracy of OD.

This study aimed to describe in detail how the optical criteria for SSL and their WASP diagnostic combinations are identified in colorectal lesions; this is also to determine what lesion characteristics increase the probability of correctly diagnosing an SSL. We also aimed to determine the diagnostic performance of the Hazewinkel's criteria depending on lesion characteristics, mainly focusing on diminutive lesions.

\section{Patients and methods}

This was a prospective study in which all individuals referred for a colonoscopy to the Valencian CRC screening program between April 2017 and October 2018 were included. This program is based on initial fecal immunochemical testing (FIT), which is followed by a colonoscopy when a positive FIT is obtained.

\section{Endoscopists}

Five endoscopists from one tertiary referral center participated in the study, and all of them were experts in colonoscopy (ADR $>60 \%$; more than 500 colonoscopies per year). They were all aware of the NICE classification but were not experts in OD. Therefore, all the participants attended a 30-minute classroom didactic training session hosted by an endoscopist who was an expert in optical diagnosis (MB). This session included an explanation about the detection of serrated lesions, a description of the WASP classification with individual comments on each optical criterion, and an explanation of the stepwise use of the NICE classification, and the optical criteria for serrated lesions. Some tips for recognizing dysplasia on a serrated lesion were also given, intended to avoid misdiagnosing an adenoma [11, 12]. Dur-
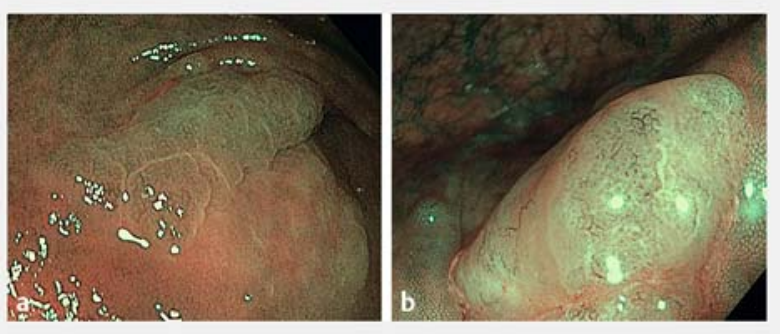

- Fig. 1 Examples of optical criteria for SSL diagnosis: a Cloud-like appearance and irregular shape. $\mathbf{b}$ Cloud-like appearance and black dots.

ing the same period of the study, the same expert in OD participated in the CRC screening program as well. His performance values were used as a benchmark for comparisons with each learner's performance, to verify the efficacy of the learning session.

\section{Colonoscopies}

All examinations were performed with high-definition endoscopes (CF-H180AL, CF-H190AL) and processors (EVIS EXERA II and EXERA III). All endoscopy suites were equipped with highdefinition LCD screens.

For all examinations, the maximum insertion depth and the quality of the examination, in total and per segment, using the Boston Bowel Preparation Scale (BBPS), were recorded. The size (measuring the width of the lesion with an open biopsy forceps), morphology (Paris classification) [13], and location of every lesion (proximal vs distal) were also collected.

\section{Optical and pathological diagnosis}

A thorough washing of every lesion was advised, to eliminate mucus and debris that may make OD difficult. Endoscopists were asked to classify each lesion using the NICE classification and then determine the presence of each one of the Hazewinkel's optical criteria for SSL. Following the initial Hazewinkel's et al. [8] description, cloud-like appearance was defined as the presence of a soft-looking nodular surface resembling a cumulus cloud; indistinctive limits were defined as a vague demarcation of the border of a lesion; irregular morphology was described as an asymmetric shape, different to the regular oval shape of conventional polyps; and black dots were described as the presence of small dark dots inside the open crypts. The presence of two or more criteria was considered diagnostic of SSL. Other known optical criteria for SSL, like the presence of a mucus cap, were not considered. No formal OD of SSL was demanded; therefore, no diagnostic confidence level was recorded. The ability to recognize dysplasia on SSL was not determined.

All lesions proximal to the rectosigmoid junction, irrespective of its optical appearance, were resected. The decision of resecting hyperplastic-looking diminutive rectal polyps was left to the endoscopist. The lesions were removed by the usual methods (biopsy forceps, cold snare, hot polypectomy, or 
endoscopic mucosal resection) at the discretion of the endoscopist. Each lesion was placed in a separate jar to allow individual pathological analysis. Three expert GI pathologists reviewed all the samples. For the pathological diagnosis of an SSL, the presence of a unique architecturally distorted, dilated and/or horizontally branched crypt, associated with inverted maturation, would suffice [14]. Before beginning the study, to unify the pathological diagnostic criteria for SSL, three voting rounds among the participant pathologists took place, in which several serrated lesions were reviewed until at least a moderate concordance between pathologists $(k=0.6)$ in the diagnosis of SSL was obtained.

The OD of the lesions was compared with that of the final pathological report to calculate diagnostic performance measures. If any lesions were lost before histopathological examination or an optical examination could not be performed, the lesion was excluded from the analysis.

\section{Statistical methods}

All data from patients, endoscopies, and lesions were prospectively included in an anonymized data base. Continuous variables were described using median, range, and SD. Categorical values were described using percentages. The frequency of every combination of OD criteria for SSL among lesions with a pathological diagnosis of SSL was recorded, overall, and related to size, location, and previous NICE classification. Lesion's size was categorized in lesions $\leq 5 \mathrm{~mm}$ (diminutive lesions), lesions between 6 and $9 \mathrm{~mm}$ and lesions $\geq 10 \mathrm{~mm}$. Regarding location, proximal lesions were considered when they were located proximal to left colon. The frequency of SSLs among lesions fulfilling the OD criteria was also recorded. The accuracy, sensitivity, specificity, positive and negative predictive values, and positive likelihood ratio were calculated, overall and for every endoscopist. A multivariable analysis was performed to show which variables (size, NICE classification, location) were related to a greater probability of correctly identifying an SSL. Because one patient can harbor several lesions and the lesions were inspected by the same endoscopist, for analysis purposes, the lesions cannot be considered as truly independent.

Therefore, a random-effects logistic regression model with distinguishable data should be used. The odds ratios (OR) were estimated from the model and given with their $95 \%$ confidence intervals $(95 \% \mathrm{Cl})$. All significance tests were two-tailed, and $P$ values $<0.05$ were considered to be statistically significant. STATA v. 14.0 software (Stata Corp., College Station, Texas, United States) was used for the data analysis.

\section{Ethical approval and role of the funding source}

The study was reviewed and approved by the medical ethical review board of the La Fe University Hospital (No.2016/0477). This is a nested study to one registered in ClinicalTrials.gov (NCT03089268). All patients provided informed consent before participating in the study. This study was partially funded by a grant from the Spanish Society for Gastrointestinal Endoscopy Foundation.

\section{Results}

In total, 438 patients, 209 women (47.7\%), median (SD) age 62.1 years (5.9) and 552 colonoscopies were included. The median BBPS was determined to be 7 (SD 1.8) [BBPS 0-5 $102(18.5 \%)]$. The cecum was reached in 520 colonoscopies (94.2\%).

\section{Polyp characteristics}

Overall, 1493 lesions were evaluated, but 97 (6.5\%) had to be excluded because of the lack of pathological diagnosis (lost sample, the sample was too small for analysis). Therefore, only 1396 lesions were suitable for comparison. Most of the lesions were adenomas (72.2\%), diminutive (64.3\%), and sessile (74.2\%). Pathological diagnosis showed an SSL in 79 lesions (5.7\%). Most of these were diminutive (43.0\%), sessile (59.5\%), and NICE type 1 (55.7\%). No SSL showed high-grade dysplasia, and 15 (19.0\%) of SSL had low grade dysplasia. The main characteristics of the lesions and the 79 SSL are presented in $>$ Table 1.

\section{Primary outcome: frequency and distribution of the optical diagnostic criteria for SSL}

The individual optical criteria identified in the SSL were cloudlike appearance in 35 (44.3\%), indistinct borders 23 (29.1\%), irregular shape 21 (26.6\%), and black dots in 10 (12.7\%). The distribution of these criteria depending on the lesion's characteristics is depicted in $\mathbf{F i g . 2}$. Overall, OD criteria were more frequently identified in NICE type 1 lesions, lesions $>10 \mathrm{~mm}$, and proximal lesions. Regarding diminutive SSL, only 10 (29.4\%) had a cloud-like appearance, five (14.7\%) had indistinct borders or irregular shape, and only two (5.9\%) had black dots (> Fig. 2).

Focusing on the optical criteria combinations that are diagnostic of SSL, only 26 of the 79 SLL fulfilled the required combinations (sensitivity 32.9\%, 95\% Cl 29.1\%-36.7\%) (> Table 2). Cloud-like appearance+irregular shape+indistinct borders and the full quartet were the most frequently detected combinations (9/79, 11.4\% each) ( $\triangleright$ Table 3 ). Cloud-like appearance was present in all diagnostic combinations, but none of these included the black dots, except for the quartet. The quartet was most frequently found in NICE type $1,>10 \mathrm{~mm}$, and proximal SSL. Only five of 34 diminutive SSLs (14.7\%) had two or more optical diagnostic criteria, none of them being the duplets ( $\triangleright$ Table 2 ).

In all, 85 lesions (6.1\%) fulfilled the Hazewinkel's criteria to be diagnosed as an SSL. However, only 26 of them were ultimately diagnosed as SSL (positive predictive value 30.6\%, $95 \%$ $\mathrm{Cl}$ 26.9-34.3) (Supplementary Table1). The combinations more predictive of SSL were cloud-like appearance + indistinct borders $(5 / 8,62.5 \%)$ and the quartet $(9 / 20,45 \%)$. In diminutive lesions, no duplet increased the probability of finding an SSL (Supplementary Table1). Size $>5 \mathrm{~mm}$ (OR 1.28, $95 \% \mathrm{Cl}$ 1.11-1.48), proximal location (OR 1.06, $95 \% \mathrm{Cl} 1.06-1.44)$, but not NICE type 1 lesions (OR 1.06, 95\% Cl-1.09-1.24) increased the probability of correctly diagnosing an SSL when a positive OD was done. 
- Table 1 Lesion characteristics.

\begin{tabular}{|c|c|c|c|c|}
\hline & \multicolumn{2}{|c|}{ All lesions ( $n=1396$ ) } & \multicolumn{2}{|c|}{ SSL $(n=79)$} \\
\hline & $\mathbf{n}$ & $(\%)$ & $\mathbf{n}$ & (\%) \\
\hline \multicolumn{5}{|l|}{ Pathology } \\
\hline - Adenoma & 1008 & $(72.2)$ & - & - \\
\hline - Hyperplastic & 216 & $(15.5)$ & - & - \\
\hline . SSL & 79 & $(5.7)$ & - & - \\
\hline . TSA & 16 & $(1.1)$ & - & - \\
\hline - Other/normal & 77 & $(5.5)$ & - & - \\
\hline \multicolumn{5}{|l|}{ Size (mm) } \\
\hline . $1-5$ & 898 & $(64.3)$ & 34 & $(43.0)$ \\
\hline - 6-9 & 325 & $(23.3)$ & 29 & $(36.7)$ \\
\hline - $10-20$ & 153 & $(11.0)$ & 15 & $(19.0)$ \\
\hline - 20-60 & 13 & $(1.0)$ & 1 & $(1.3)$ \\
\hline \multicolumn{5}{|l|}{ Morphology } \\
\hline . 0-Ip & 132 & $(9.5)$ & 1 & $(1.3)$ \\
\hline . 0 -Is & 1036 & $(74.2)$ & 47 & (59.5) \\
\hline - 0-IIa & 181 & $(13.0)$ & 18 & $(22.8)$ \\
\hline . 0-IIb & 15 & $(1.1)$ & 8 & $(10.1)$ \\
\hline . $0-\|l a+\| c$ & 1 & $(0.07)$ & - & - \\
\hline . LST & 19 & $(1.4)$ & 5 & $(6.4)$ \\
\hline \multicolumn{5}{|l|}{ Location } \\
\hline - Proximal & 742 & $(53.1)$ & 45 & $(57.0)$ \\
\hline . Distal & 651 & $(46.6)$ & 34 & $(43.0)$ \\
\hline \multicolumn{5}{|l|}{ NICE } \\
\hline .1 & 335 & $(24.0)$ & 44 & $(55.7)$ \\
\hline .2 & 963 & $(69.0)$ & 29 & $(36.7)$ \\
\hline .3 & 17 & $(1.2)$ & 1 & $(1.3)$ \\
\hline - Undefined & 11 & $(0.8)$ & & \\
\hline
\end{tabular}

\section{Secondary outcome: diagnostic performance of Hazewinkel's criteria}

The overall accuracy of the OD criteria for an SSL diagnosis was $92.0 \%, 95 \% \mathrm{Cl} 89.8 \%-94.2 \%$. Overall accuracy was found to be significantly worse for NICE1 lesions than for NICE2 lesions (84.2\%, 95\% Cl 81.1\%-86.9\% vs $94.8 \%$, $95 \%$ Cl 93.3\%-96.7\%). However, sensitivity and PPV were higher for NICE type 1 lesions (sensitivity $43.2 \%, 95 \% \mathrm{Cl} 39.2 \%-47.2 \%$ vs $23.3 \%$, $95 \%$ Cl $19.9 \%-26.7 \%$ and PPV $40.4 \%, 95 \% \mathrm{Cl} 36.5 \%-44.4 \%$ vs $20.0 \%, 95 \% \mathrm{Cl} 16.8 \%-23.2 \%)$. Sensitivity and PPV were also higher for $>10 \mathrm{~mm}$ and proximal lesions. Regarding both di-

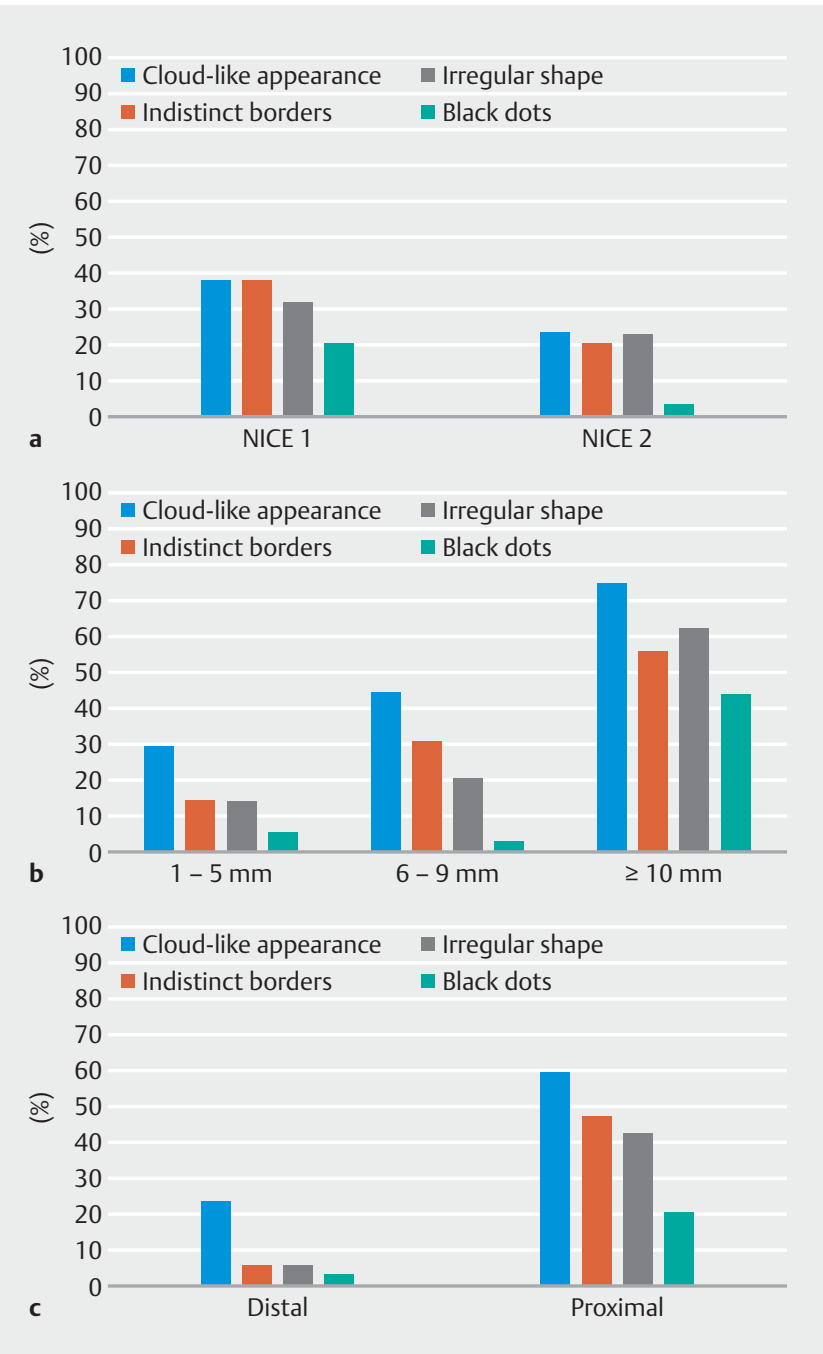

- Fig. 2 Frequency of each optical criterion for SSL depending on lesion characteristics. a NICE classification. b Size. c Location.

minutive and distal lesions, sensitivity and PPV dropped significantly ( $>$ Table 3 ).

\section{Quality of learning assessment}

No significant differences between endoscopists in diagnostic performance measures were detected. During the same period, an examiner, who is an expert in OD, performed 168 colonoscopies, examining 570 lesions. The learner's performance measures were similar to those of the expert in OD (endoscopist no. 6) (Supplementary Table2). On the other hand, the overall negative predictive value (NPV) for neoplastic (adenoma $+\mathrm{SSL}$ ) diminutive distal lesions was determined to be at $94.25 \%$.

\section{Discussion}

Our study shows that the accuracy of the Hazewinkel's criteria in providing a positive diagnosis of SSL in real colonoscopies is insufficient. Only 29 of 79 lesions (32.9\%, $95 \%$ Cl 29.1\%-36.7\%) with a pathological diagnosis of SSL had two or more OD criteria. In addition, only 26 lesions (30.6\%, $95 \%$ Cl 26.9\%-34.3\%) 
- Table2 Distribution of optical diagnostic criteria in lesions with a final pathological diagnosis of SSL.

\begin{tabular}{|c|c|c|c|c|c|c|c|c|c|c|c|c|c|c|c|c|}
\hline & \multicolumn{2}{|c|}{$\begin{array}{l}\text { Overall } \\
(n=79)\end{array}$} & \multicolumn{2}{|c|}{$\begin{array}{l}\text { NICE type } 1 \\
(n=44)\end{array}$} & \multicolumn{2}{|c|}{$\begin{array}{l}\text { NICE type } 2 / 3 \\
(n=29)\end{array}$} & \multicolumn{2}{|c|}{$\begin{array}{l}1-5 \mathrm{~mm} \\
(\mathrm{n}=34)\end{array}$} & \multicolumn{2}{|c|}{$\begin{array}{l}6-9 \mathrm{~mm} \\
(\mathrm{n}=29)\end{array}$} & \multicolumn{2}{|c|}{$\begin{array}{l}>10 \mathrm{~mm} \\
(\mathrm{n}=16)\end{array}$} & \multicolumn{2}{|c|}{$\begin{array}{l}\text { Proximal } \\
(n=45)\end{array}$} & \multicolumn{2}{|c|}{$\begin{array}{l}\text { Distal } \\
(n=651)\end{array}$} \\
\hline & $n$ & (\%) & $n$ & (\%) & $n$ & (\%) & $\mathrm{n}$ & (\%) & $n$ & (\%) & $n$ & (\%) & $n$ & (\%) & $\mathbf{n}$ & (\%) \\
\hline $\begin{array}{l}\text { Cloud-like } \\
\text { appearance } \\
+ \text { +irregular } \\
\text { shape }\end{array}$ & 3 & (3.8) & 2 & $(4.55)$ & 1 & (3.4) & 0 & - & 1 & (3.4) & 2 & (12.5) & 3 & (6.7) & 0 & - \\
\hline $\begin{array}{l}\text { Cloud-like } \\
\text { appearance } \\
\text { + indistinct } \\
\text { borders }\end{array}$ & 5 & (6.3) & 5 & (11.4) & 1 & $(0.10)$ & 0 & - & 4 & (13.8) & 1 & (6.2) & 5 & (11.1) & 0 & - \\
\hline $\begin{array}{l}\text { Cloud-like } \\
\text { appearance } \\
\text { +black dots }\end{array}$ & 0 & - & 0 & - & 0 & - & 0 & - & 0 & - & 0 & - & 0 & - & 0 & - \\
\hline $\begin{array}{l}\text { Irregular } \\
\text { shape + in- } \\
\text { distinct bor- } \\
\text { ders }\end{array}$ & 0 & & 0 & - & 0 & - & 0 & - & 0 & - & 0 & - & 0 & - & 0 & - \\
\hline $\begin{array}{l}\text { Indistinct } \\
\text { borders + } \\
\text { black dots }\end{array}$ & 0 & - & 0 & - & 0 & - & 0 & - & 0 & - & 0 & - & 0 & - & 0 & - \\
\hline $\begin{array}{l}\text { Irregular } \\
\text { shape + } \\
\text { black dots }\end{array}$ & 0 & - & 0 & - & 0 & - & 0 & - & 0 & - & 0 & - & 0 & - & 0 & - \\
\hline $\begin{array}{l}\text { Cloud-like } \\
\text { appearance } \\
+ \text { +irregular } \\
\text { shape + in- } \\
\text { distinct bor- } \\
\text { ders }\end{array}$ & 9 & (11.4) & 4 & (9.1) & 5 & $(17.2)$ & 4 & (11.8) & 4 & (13.8) & 1 & (6.2) & 7 & (15.6) & 2 & (5.9) \\
\hline $\begin{array}{l}\text { Cloud-like } \\
\text { appearance } \\
+ \text { +irregular } \\
\text { shape }+ \\
\text { black dots }\end{array}$ & 0 & - & 0 & - & 0 & - & 0 & & 0 & - & 0 & - & 0 & - & 0 & - \\
\hline $\begin{array}{l}\text { Irregular } \\
\text { shape + in- } \\
\text { distinct bor- } \\
\text { ders + black } \\
\text { dots }\end{array}$ & 0 & - & 0 & - & 0 & - & 0 & & 0 & - & 0 & - & 0 & - & 0 & - \\
\hline $\begin{array}{l}\text { Cloud-like } \\
\text { appearance } \\
\text { + irregular } \\
\text { shape + in- } \\
\text { distinct bor- } \\
\text { ders + black } \\
\text { dots }\end{array}$ & 9 & (11.4) & 8 & (18.2) & 1 & (3.4) & 1 & (2.94) & 1 & (3.4) & 7 & (43.7) & 9 & $(20.0)$ & 0 & - \\
\hline Total & 26 & (32.9) & 19 & $(43.2)$ & 7 & $(24.1)$ & 5 & (14.7) & 10 & (34.5) & 11 & (68.7) & 24 & (53.3) & 2 & (5.9) \\
\hline
\end{tabular}

SSL, sessile serrated lesion; NICE, NBI International Colorectal Endoscopic Classification.

fulfilling the WASP classification criteria for an SSL eventually got that diagnosis. This situation is even worse for diminutive lesions (sensitivity $14.7 \%$, $95 \%$ Cl 11.9\%-17.6\%; PPV 14.3\%, $95 \%$ Cl $11.5 \%-17.1 \%$ ) ( Table 3 ). Most of the diminutive SSL lacked the optical criteria ( $>$ Fig. 2 ).
Several studies have evaluated the sensitivity of SSL OD, most of them using NBI plus magnification, showing values ranging between $56 \%$ and $92 \%$ [15-19]. Despite the heterogeneity among studies, mainly due to different optical criteria for the identification of SSL, different types of lesions evaluated, 
- Table 3 Performance measures for optical diagnosis of SSL depending on lesion characteristics.

\begin{tabular}{|c|c|c|c|c|c|c|c|c|}
\hline & $\begin{array}{l}\text { Overall } \\
(n=1396)\end{array}$ & $\begin{array}{l}\text { NICE type } 1 \\
(n=335)\end{array}$ & $\begin{array}{l}\text { NICE type } 2 / 3 \\
(n=980)\end{array}$ & $\begin{array}{l}1-5 \mathrm{~mm} \\
(\mathrm{n}=898)\end{array}$ & $\begin{array}{l}6-9 \mathrm{~mm} \\
(\mathrm{n}=325)\end{array}$ & $\begin{array}{l}>10 \mathrm{~mm} \\
(\mathrm{n}=166)\end{array}$ & $\begin{array}{l}\text { Proximal } \\
(n=742)\end{array}$ & $\begin{array}{l}\text { Distal } \\
(n=651)\end{array}$ \\
\hline Acc & $\begin{array}{l}92.0 \\
(89.8-94.2)\end{array}$ & $\begin{array}{l}84.2 \\
(81.1-86.9)\end{array}$ & $\begin{array}{l}94.8 \\
(93.3-96.7)\end{array}$ & $\begin{array}{l}93.4 \\
(91.0-95.0)\end{array}$ & $\begin{array}{l}89.5 \\
(86.5-91.5)\end{array}$ & $\begin{array}{l}88.5 \\
(85.4-90.6)\end{array}$ & $\begin{array}{l}91.9 \\
(89.8-94.2)\end{array}$ & $\begin{array}{l}92.0 \\
(89.9-94.2)\end{array}$ \\
\hline$S$ & $\begin{array}{l}32.9 \\
(29.1-36.7)\end{array}$ & $\begin{array}{l}43.2 \\
(39.2-47.2)\end{array}$ & $\begin{array}{l}23.3 \\
(19.9-26.7)\end{array}$ & $\begin{array}{l}14.7 \\
(11.9-17.6)\end{array}$ & $\begin{array}{l}34.5 \\
(30.7-38.3)\end{array}$ & $\begin{array}{l}68.7 \\
(65.0-72.5)\end{array}$ & $\begin{array}{l}53.3 \\
(49.3-57.3)\end{array}$ & $\begin{array}{l}5.9( \\
4.0-7.8)\end{array}$ \\
\hline $\mathrm{E}$ & $\begin{array}{l}95.5 \\
(93.9-97.2)\end{array}$ & $\begin{array}{l}90.4 \\
(88.0-92.7)\end{array}$ & $\begin{array}{l}97.0 \\
(95.7-98.4)\end{array}$ & $\begin{array}{l}96.5 \\
(95.1-98.0)\end{array}$ & $\begin{array}{l}94.9 \\
(93.2-96.7)\end{array}$ & $\begin{array}{l}90.7 \\
(88.3-93.0)\end{array}$ & $\begin{array}{l}94.4 \\
(92.6-96.2)\end{array}$ & $\begin{array}{l}96.8 \\
(95.3-96.2)\end{array}$ \\
\hline PPV & $\begin{array}{l}30.6 \\
(26.9-34.3)\end{array}$ & $\begin{array}{l}40.4 \\
(36.5-44.4)\end{array}$ & $\begin{array}{l}20.0 \\
(16.8-23.2)\end{array}$ & $\begin{array}{l}14.3 \\
(11.5-17.1)\end{array}$ & $\begin{array}{l}40.0 \\
(36.1-43.9)\end{array}$ & $\begin{array}{l}44.0 \\
(40.0-48.0)\end{array}$ & $\begin{array}{l}38.1 \\
(34.2-42.0)\end{array}$ & $\begin{array}{l}9.1 \\
(5.8-10.2)\end{array}$ \\
\hline NPV & $\begin{array}{l}95.6 \\
(93.9-97.2)\end{array}$ & $\begin{array}{l}91.3 \\
(89.1-93.6)\end{array}$ & $\begin{array}{l}97.6 \\
(96.3-98.8)\end{array}$ & $\begin{array}{l}96.7 \\
(95.2-98.1)\end{array}$ & $\begin{array}{l}93.7 \\
(91.7-95.6)\end{array}$ & $\begin{array}{l}96.4 \\
(95.0-97.3)\end{array}$ & $\begin{array}{l}96.9 \\
(95.5-98.3)\end{array}$ & $\begin{array}{l}94.9 \\
(92.1-95.9)\end{array}$ \\
\hline $\mathrm{LR}^{+}$ & $\begin{array}{l}7.35 \\
(6.4-8.4)\end{array}$ & $\begin{array}{l}4.5 \\
(3.8-5.3)\end{array}$ & $\begin{array}{l}7.9 \\
(6.1-10.2)\end{array}$ & $\begin{array}{l}4.2 \\
(3.1-5.7)\end{array}$ & $\begin{array}{l}6.8 \\
(5.3-8.7)\end{array}$ & $\begin{array}{l}7.4 \\
(6.0-9.0)\end{array}$ & $\begin{array}{l}9.5 \\
(8.3-11.0)\end{array}$ & $\begin{array}{l}1.8 \\
(1.1-2.9)\end{array}$ \\
\hline
\end{tabular}

NICE, NBI International Colorectal Endoscopic Classification; Acc, accuracy; S, sensitivity; E, specificity; PPV, positive predictive value; NPV, negative predictive value; $\mathrm{LR}+$, positive likelihood ratio; data are given as\% $(95 \% \mathrm{Cl})$.

and disparate designs [20], it seems that magnification may be partially responsible for these higher sensitivity values. With magnification, other optical characteristics like vascular and pit patterns become apparent, adding information that can contribute in making a more accurate diagnosis. Equivalent $\mathrm{NBI}$ studies without magnification are scarce, most coming from the Dutch group, the designers of the WASP classification. A retrospective study on still pictures, using the criteria later incorporated into the WASP classification, found a sensitivity of $89 \%$ [8]. However, two studies evaluated WASP classification in real colonoscopies, showing a sensitivity of $42 \%$ and $74 \%$ respectively, but only for diminutive lesions diagnosed with high confidence $[10,21]$. In one study, including diminutive lesions and small polyps, the overall sensitivity was determined to be $53.3 \%$, and the sensitivity for high-confidence diagnosis was $56 \%$ [22]. In our study, as no specific diagnosis of SSL was provided, the confidence in SSL diagnosis was not recorded. This may partially explain our relatively low sensitivity values, but a lack of reproducibility, being the only such study not performed by the WASP developers, cannot be excluded. If positive SSL diagnosis can be improved using pseudo-magnification systems like dual-focus from Olympus remains to be demonstrated.

Even less information is available about the specific performance of each of Hazewinkel's criteria. In our study, the most frequently found criterion was cloud-like appearance, which was present in all diagnostic combinations. On the other hand, in our sample, black dots, either alone or in combination with other features, were only found in $12.7 \%$ of all lesions and were never identified in SSL except for the quartet. Size may have had some influence on this result because black dots were more frequently identified in lesions $\geq 10 \mathrm{~mm}$ than in smaller lesions ( $4.8 \%$ vs. $43.7 \%, P=0.0001$ ), and only $12 \%$ of our sample was $\geq 10 \mathrm{~mm}$ lesions. The presence of a mucus cap may also hamper the identification of black dots. However, in our protocol, every lesion was washed free of mucus and debris before performing OD. Moreover, mucin seems more prevalent in larger lesions [23], and, in our study, black dots were identified more easily in such lesions, going against a significant influence of mucus cap on the low prevalence of black dots in SSLs. The absence of magnification could have also played a role. Studies with magnification find black dots (expanded crypt openings, pit pattern II-O) between $26 \%$ and $84 \%$ of SSL [15-17,23]. However, even with magnification, the II-O pattern has been observed to change with time along with changes in the shape of the lesion and the amount of mucus [24].

Despite these low values of sensitivity, the overall accuracy was high $(92.0 \%, 95 \% \mathrm{Cl} 89.9 \%-94.2 \%)$, due to a good capacity to deal with lesions other than SSL. The specificity was $95.5 \%$, $95 \% \mathrm{Cl} 93.9 \%-97.2 \%$, and NPV was $95.6 \%$, 95\% Cl 93.9\%$97.2 \%$. Again, heterogeneity makes comparisons difficult. Many $\mathrm{NBI}+$ magnification studies aimed to differentiate SLL from hyperplastic polyps, and, as a consequence, the accuracy was lower, ranging from $60 \%$ to $80 \%$ depending on the OD criterion being evaluated [15-17]. In the Dutch CRC screening program, the overall accuracy and NPV using the WASP classification on small polyps were shown to be $86.8 \%$ and $96.9 \%$ [22], which was similar to ours. The aforementioned study of Vleugels et al. [10] showed an overall accuracy of $97.2 \%$ for diminutive proximal lesions, but again only for high-confidence diagnosis.

The lesion characteristics influenced performance. Accuracy was better for NICE type 2 lesions $(94.8 \%$, $95 \%$ Cl 93.3\%$96.7 \%$ ) vs $84.2 \%$, $95 \% \mathrm{Cl} 81.1 \%-86.9 \%)$. However, sensitivity was better for NICE type 1 lesions $(43.2 \%$, 95\% Cl 39.2\%$47.2 \%$ ), lesions $\geq 10 \mathrm{~mm}$ (68.7\%, $95 \% \mathrm{Cl} 65.0 \%-72.5 \%)$, and proximal lesions (53.3\%, $95 \% \mathrm{Cl} 49.3 \%-57.3 \%$ ). Similar results were obtained for PPV. In multivariate analysis, the two main characteristics related to a greater probability of a correct SSL diagnosis were size $\geq 5 \mathrm{~mm}$ and proximal location. Other studies have also identified size $\geq 10 \mathrm{~mm}$ and proximal location as risk factors for SSL [18]. Results concerning the relationship 
between OD and NICE are scarce because many studies do not describe the NICE status [15] or are performed exclusively in NICE type 1 lesions [17].

In our sample of diminutive lesions, which comprised 34 of the 79 SSLs (43\%), the accuracy was similar to that seen with larger lesions, but the sensitivity and PPV were much lower (14.7\%, 95\% Cl 11.9-17.6) and 14.3\%, 95\% Cl 11.5-17.1) respectively, ( $>$ Table 3 ). Only $9 \%$ of proximal and $25 \%$ of distal diminutive SSL were correctly identified. This may be due to the low prevalence of Hazewinkel's criteria in diminutive lesions. The most prevalent criterion of the OD combination was cloud-like appearance, and this was only present in $29.4 \%$ of cases. These values are much lower than the ones reported by Vleugels et al. [10] who showed a sensitivity and PPV of $52.4 \%$ and $40.7 \%$ respectively, for diminutive proximal lesions diagnosed with high confidence. However, in this study, only $59.5 \%$ of diminutive lesions were diagnosed with high confidence, a number much higher than recommended when applying the NICE classification. A very recent study from the same group showed a $74 \%$ sensitivity in diminutive lesions diagnosed with high confidence. However, participant endoscopists were a selected group who had to fulfill quality parameters to participate in the trial, and the percentage of lesions diagnosed with high confidence was not provided [21].

The low accuracy in providing a positive diagnosis of SLL may be of importance when it comes to applying the resect and discard strategy because some diminutive neoplastic lesions will be missed. Other approaches may be warranted to deal with diminutive lesions in a resect and discard background, for example, by finding ways to improve the positive OD of SSL or considering all proximal lesions as adenomas [25].

This study had some limitations. First, probably because of our strict pathological criteria, only 79 SSLs (34 diminutive) were included, a relatively small sample. However, the studies describing the Hazewinkel's criteria and the WASP classification included a similar number of SSLs [8,9] and so did most of the magnification studies evaluating OD of SSLs [15]. Second, it was developed with a learning background, with participant endoscopists not being expert in OD. However, they were experienced endoscopists, and they used the NICE classification daily. Following the teaching session, their diagnostic performance values were similar and also were equivalent to that of an endoscopist expert in OD. Moreover, the final NPV for distal neoplastic lesions of the whole group of endoscopists was 94.2, fulfilling the second PIVI criteria [5]. Third, since the main aim of the study was to describe the behavior of the optical diagnostic criteria for SSL, a confidence level on a final diagnosis of SSL was not required. Therefore, diagnostic performance values may be lower than in other reports that considered confidence levels. Fourth, this is a single-center study, which may hamper extrapolation of results. However, the strict criteria used for the design may ease reproducibility, provided that experienced endoscopists perform the examinations.

The study had some strengths. First, because the pathological review of serrated polyps tends to have a high interobserver variability [26], we performed some diagnostic rounds among our specialized gastrointestinal pathologist to achieve a moder- ate to high level of concordance before beginning the study. Second, our study population was consecutive FIT-positive patients from our CRC screening program, providing a homogeneous sample of patients.

\section{Conclusion}

In conclusion, Hazewinkel's criteria for OD of SSL are not effective for a positive diagnosis, particularly in the case of diminutive lesions. Most confirmed SSLs lacked the OD criteria. Although diagnostic ability improves with size and proximal location, it may not be accurate enough for reliable identification of SSL. Different approaches to improve the OD of SSL are warranted.

\section{Acknowlegements}

This study was supported by a grant from the Spanish Society of Gastrointestinal Endoscopy Foundation (03-2017). Partial results of this research were published in abstract form at the ESGE Days 2019 meeting, held in Prague.

\section{Competing interests}

The authors declare that they have no conflict of interest.

\section{References}

[1] Snover DC. Update on the serrated pathway to colorectal carcinoma. Hum Pathol 2011; 42: 1-10

[2] Satorres C, Garcia-Campos M, Bustamante-Balen M. Molecular features of the serrated pathway to colorectal cancer: current knowledge and future directions. Gut Liver 2020. doi:10.5009/gnl19402

[3] Pohl H, Srivastava A, Bensen SP et al. Incomplete polyp resection during colonoscopy-results of the complete adenoma resection (CARE) study. Gastroenterology 2013; 144: 74-80 e71

[4] Oono Y, Fu K, Nakamura $\mathrm{H}$ et al. Progression of a sessile serrated adenoma to an early invasive cancer within 8 months. Digest Dis Sci 2009; 54: 906-909

[5] Rex DK, Kahi C, O'Brien M et al. The American Society for Gastrointestinal Endoscopy PIVI (Preservation and Incorporation of Valuable Endoscopic Innovations) on real-time endoscopic assessment of the histology of diminutive colorectal polyps. Gastrointest Endosc 2011; 73: 419-422

[6] Ignjatovic A, East JE, Suzuki N et al. Optical diagnosis of small colorectal polyps at routine colonoscopy (Detect InSpect ChAracterise Resect and Discard; DISCARD trial): a prospective cohort study. Lancet Oncol 2009; 10: 1171-1178

[7] Terdiman JP, McQuaid KR. Surveillance guidelines should be updated to recognize the importance of serrated polyps. Gastroenterology 2010; 139: 1444-1447

[8] Hazewinkel Y, Lopez-Ceron M, East JE et al. Endoscopic features of sessile serrated adenomas: validation by international experts using high-resolution white-light endoscopy and narrow-band imaging. Gastrointest Endosc 2013; 77: 916-924

[9] JE IJ, Bastiaansen BA, van Leerdam ME et al. Development and validation of the WASP classification system for optical diagnosis of adenomas, hyperplastic polyps and sessile serrated adenomas/polyps. Gut 2016; 65: 963-970 
[10] Vleugels JLA, Dijkgraaf MGW, Hazewinkel Y et al. Effects of training and feedback on accuracy of predicting rectosigmoid neoplastic lesions and selection of surveillance intervals by endoscopists performing optical diagnosis of diminutive polyps. Gastroenterology 2018; 154: 1682-1693 e1681

[11] Bouwens MW, van Herwaarden YJ, Winkens B et al. Endoscopic characterization of sessile serrated adenomas/polyps with and without dysplasia. Endoscopy 2014; 46: 225-235

[12] Burgess NG, Pellise M, Nanda KS et al. Clinical and endoscopic predictors of cytological dysplasia or cancer in a prospective multicentre study of large sessile serrated adenomas/polyps. Gut 2016; 65: 437446

[13] The Paris endoscopic classification of superficial neoplastic lesions: esophagus, stomach, and colon: November 30 to December 1, 2002. Gastrointest Endosc 2003; 58: S3-43

[14] Rex DK, Ahnen DJ, Baron JA et al. Serrated lesions of the colorectum: review and recommendations from an expert panel. Am J Gastroenterol 2012; 107: 1315-1329; quiz 1314, 1330

[15] Yamada M, Sakamoto T, Otake Y et al. Investigating endoscopic features of sessile serrated adenomas/polyps by using narrow-band imaging with optical magnification. Gastrointest Endosc 2015; 82: 108-117

[16] Nakao Y, Saito S, Ohya T et al. Endoscopic features of colorectal serrated lesions using image-enhanced endoscopy with pathological analysis. Eur J Gastroenterol Hepatol 2013; 25: 981-988

[17] Yamashina T, Takeuchi Y, Uedo $N$ et al. Diagnostic features of sessile serrated adenoma/polyps on magnifying narrow band imaging: a prospective study of diagnostic accuracy. J Gastroenterol Hepatol 2015; 30: 117-123
[18] Uraoka T, Higashi R, Horii J et al. Prospective evaluation of endoscopic criteria characteristic of sessile serrated adenomas/polyps. J Gastroenterol 2015; 50: 555-563

[19] Singh R, Jayanna M, Navadgi S et al. Narrow-band imaging with dual focus magnification in differentiating colorectal neoplasia. Digest Endosc 2013; 25: (Suppl. 02): 16-20

[20] Parikh ND, Chaptini L, Njei B et al. Diagnosis of sessile serrated adenomas/polyps with image-enhanced endoscopy: a systematic review and meta-analysis. Endoscopy 2016; 48: 731-739

[21] Bronzwaer MES, Vleugels JLA, van Doorn SC et al. Are adenoma and serrated polyp detection rates correlated with endoscopists' sensitivity of optical diagnosis? Endoscopy 2020: doi:10.1055/a-1151-8691

[22] Vleugels JLA, Hazewinkel Y, Dijkgraaf MGW et al. Optical diagnosis expanded to small polyps: post-hoc analysis of diagnostic performance in a prospective multicenter study. Endoscopy 2019; 51: 244252

[23] Ishigooka S, Nomoto M, Obinata $\mathrm{N}$ et al. Evaluation of magnifying colonoscopy in the diagnosis of serrated polyps. World J Gastroenterol 2012; 18: 4308-4316

[24] Matsushita HO, Yamano HO. What is type II-open pit pattern? Digest Endosc 2016; 28: 60

[25] Atkinson NS, East JE. Optical biopsy and sessile serrated polyps: Is DISCARD dead? Long live DISCARD-lite! Gastrointestinal endoscopy 2015; 82: 118-121

[26] Bustamante-Balen M, Bernet L, Cano R et al. Assessing the reproducibility of the microscopic diagnosis of sessile serrated adenoma of the colon. Rev Esp Enferm Dig 2009; 101: 258-264 